\title{
The electronic structure of TEMPO, its cation and anion
}

\author{
D. Kubala ${ }^{\mathrm{a}}$, K. Regeta ${ }^{\mathrm{a}}$, R. Janečkováa ${ }^{\text {, J. Fedor }}{ }^{\mathrm{a}}, \mathrm{S}$. Grimme ${ }^{\mathrm{b}}$, A. Hansen ${ }^{\mathrm{b}}$, P. Nesvadba ${ }^{\mathrm{c}}$ and M. Allan ${ }^{\mathrm{a}, *}$ \\ ${ }^{a}$ Département de Chimie, Université de Fribourg, Fribourg, Switzerland; ${ }^{b}$ Mulliken Center for Theoretical Chemistry, Institut für \\ Physikalische und Theoretische Chemie der Universität Bonn, Bonn, Germany; ${ }^{c} B A S F$ Schweiz AG, Basel, Switzerland
}

\begin{abstract}
The electronic structure of TEMPO (2,2,6,6-Tetramethylpiperidine-N-oxyl) and its cation and anion were studied experimentally using the electron spectroscopy techniques, dissociative electron attachment (DEA) spectroscopy, electron energy-loss spectroscopy, measurement of elastic and vibrational excitation (VE) cross sections and HeI photoelectron spectroscopy. The experiments were supplemented by quantum-chemical calculations of excitation energies, ionisation potential and the Franck-Condon profile of the first photoelectron band. Electron energy-loss spectra were recorded up to $12 \mathrm{eV}$ and revealed a number of bands that were assigned to two valence and a number of Rydberg transitions. VE cross sections reveal a broad band in the 3-12 eV range, assigned to $\sigma^{*}$ shape resonances and signals in the $0-1 \mathrm{eV}$ range, assigned to a shape resonance corresponding to a temporary capture of the incident electron in the (already singly occupied) $\pi^{*}$ orbital. Narrow threshold peaks in the VE cross sections are assigned to dipole-bound resonances. The major DEA fragment was found to be $\mathrm{O}^{-}$, with bands at 5.0 and $6.87 \mathrm{eV}$, assigned to core excited resonances.
\end{abstract}

Keywords: TEMPO; electron energy-loss spectroscopy; EELS; photoelectron spectroscopy; dissociative electron attachment; TDDFT calculations; Rydberg states

\section{Introduction}

The study of processes induced by electron collisions with radicals in general is motivated by the copious presence of the latter as transient species in natural and technological plasmas. The transient nature of the radicals makes their study difficult because they are hard to prepare in sufficient density and purity. The difficulties encountered in the study of transient molecules are illustrated by the major effort required by the recent measurements of the carbene $\mathrm{CF}_{2}$ [1]. An alternative method of learning about general principles which may be present in electron-induced processes in radicals is to use stable radicals like NO [2]. The main purpose of the present work is to adopt this strategy using another stable radical, the TEMPO (2,2,6,6-Tetramethylpiperidine$\mathrm{N}$-oxyl) molecule, as a target.

Additional motivation for the present study stems from the diverse uses of the TEMPO moiety in many areas of research. Chemistry and applications of TEMPO and other nitroxide radicals have been reviewed in several monographs [3-5]. Important are applications connected with electron capture or transfer. Thus, the recently emerged organic radical battery $[6,7]$ is based on the reversible electrochemical oxidation-reduction of TEMPO and similar nitroxides $[8,9]$. Redox reactions of TEMPO and its derivatives may be useful in dye-sensitised solar cells [10] or for overchargeprotection of lithium ion batteries [11]. TEMPO-induced electron transfer quenching of fluorescence may lead to radical sensors [12]. Knowledge of the electronic structure of TEMPO is useful for understanding its reactivity [13]. It is used in gas-phase radical initiated peptide sequencing, an alternative to electron capture and electron transfer dissociation [14]. Finally, TEMPO is used in nitroxide-mediated living radical polymerisation [15-17] and as a structural probe in electron spin resonance spectroscopy [4]. A new TEMPO analogue made conveniently from so-called frustrated Lewis pairs and NO has recently been characterised [18].

In this paper, we report a study of the electronic structure of TEMPO and of its cation and anion, and its electron-induced fragmentation, by the electron spectroscopy techniques, dissociative electron attachment (DEA) spectroscopy, electron energy-loss (EEL) spectroscopy, measurement of elastic and vibrational excitation (VE) cross sections, and HeI photoelectron spectroscopy. The interpretation of the experiments is guided by quantumchemical calculations.

\section{Methods}

\subsection{Experiments}

The absolute dissociative electron attachment cross sections were measured with the instrument described in Refs. $[19,20]$, operated in the time-of-flight (TOF) mode. The absolute calibration was against the $4.4 \mathrm{eV}$ band of $\mathrm{O}^{-}$

*Corresponding author. Email: michael.allan@unifr.ch 
production from $\mathrm{CO}_{2}$, for which the cross section of 14.0 $\mathrm{pm}^{2}$ was used as in our previous work. The error of the present relative measurement is taken as $\pm 25 \%$. The energy scale calibration was against the $\mathrm{O}^{-} / \mathrm{CO}$ peak with a threshold energy of $9.63 \mathrm{eV}$.

The TOF data were complemented by recording relative spectra with a trochoidal electron monochromator and a quadrupole mass spectrometer (QMS) [21,22]. In this instrument, ions created by DEA in a target chamber, at a temperature of $358 \mathrm{~K}$, are drawn out through a hole in the target chamber by a three-element lens and focused onto a QMS. The energy scale was calibrated by measuring the $\mathrm{O}^{-}$yield from a TEMPO-carbon monoxide mixture.

The energy-loss and VE measurements were performed with a spectrometer using hemispherical analysers $[23,24]$. Absolute values of the cross sections were determined by the relative flow technique and normalised to a theoretical helium elastic cross section as described earlier. The confidence limit for the magnitudes of the inelastic cross sections is about $\pm 30 \%$. The photoelectron spectrum (PES) was recorded with a modified Perkin Elmer PS18 HeI photoelectron spectrometer. TEMPO, from BASF Schweiz $\mathrm{AG}, \mathrm{mp} 37^{\circ}-39^{\circ}$, pure by thin layer chromatography, was used without further purification. The sample had enough vapour pressure to be kept at room temperature during all measurements.

\subsection{Theory}

The ground-state $\left({ }^{2} A^{\prime}\right)$ structure of TEMPO was optimised in $C_{S}$ symmetry with the Perdew-Burke-Ernzerhof zeroparameter global hybrid functional (PBE0) [25,26], employing the def-TZVP Gaussian AO basis set [27,28] and the well-established D3(BJ) dispersion correction scheme proposed by one of the authors (S.G.) $[29,30]$. These and the TDDFT [31,32] and approximate coupled-cluster secondorder (CC2 $[33,34])$ calculations of the excitation energies and transition moments are conducted at the spinunrestricted level using the TURBOMOLE code [35]. Core orbitals were excluded in general from the correlation treatments. The basis set was augmented by additional polarisation and diffuse functions in the excited-state calculations according to the prescription of Furche [36] (dubbed def2TZVPD). All values refer to vertical transitions employing the ground-state geometry except for the first ionisation energy for which the structure of the ion state was also optimised at the above-mentioned PBE0-D3/def-TZVP level in order to obtain the corresponding adiabatic (relaxed) and $0-0$ value. The employed harmonic frequencies were computed analytically at the PBE0-D3/def-TZVP level and scaled by 0.96 . The computation of the Franck-Condon (FC) factors for the first PES band was conducted as described in detail in Ref. [37,38] The first doublet-quartet excitation energy with DFT was computed at the SCF level as the difference of $A^{\prime}, S=1 / 2, A^{\prime}, S=3 / 2$ and $A^{\prime \prime}, S=3 / 2$ state energies.

The (strongly-contracted) $\mathrm{N}$-electron valence secondorder perturbation theory (NEVPT2 [39]) calculations for comparison were conducted with the ORCA code $[40,41]$. Quasi-restricted orbitals ([42]; obtained with PBE0/def2TZVP) and a complete active space with five electrons in six orbitals (CAS $(5,6)$; four roots) were used. The NEVPT2 excitation energies for the doublet state were obtained with the same def2-TZVPD basis set as in the time-dependent calculations, while the values for the quartet state refer to a state-averaged NEVPT2 calculation (doublet/quartet; four roots each with equal weight) using the smaller def2-TZVP basis set.

Subsidiary calculations of vibrational frequencies and anion energies were performed with the B3LYP functional using the Firefly code [43], based partly on GAMESS [44], and the Gaussian 09 code [45].

\section{Results and discussion}

\subsection{Positive ion}

Our HeI PES, shown as the top trace in Figure 1, agrees well with that of Novak et al. [46]. Our experimental values of the ionisation energies are indicated in Figure 1 and are accurate within $\pm 30 \mathrm{meV}$. The calculations of Novak et al. led to an unambiguous assignment of the first three bands in the spectrum - indicated in Figure 1 - and we therefore do not present further calculations on the cation, except the lowest adiabatic and vertical ionisation energies given in Table 1. The electronic configurations are shown in Figure 2. The nitroxide group is isoelectronic with the radical anion of a carbonyl group. The first ionisation is thus from the $\pi^{*}$ orbital, leading to the ${ }^{1} A^{\prime}$ cation state. Next ionisation is from the $n$ orbital, leading to the ${ }^{3} A^{\prime \prime}$ and ${ }^{1} A^{\prime \prime}$ pair of states.

Our spectrum shows weak vibrational structure in the first band, with a spacing of about $0.19 \mathrm{eV}(1530 \pm$ $150 \mathrm{~cm}^{-1}$ ) which would correspond to the expected NO stretch vibration. To gain a deeper understanding of the structure, we calculated the FC profile of this band and compare it with the experiment in Figure 3. The calculated spectrum is shown convoluted by a simulated instrumental width of $250 \mathrm{~cm}^{-1}$ for comparison with the experiment, but also with a $10 \mathrm{~cm}^{-1}$ width to show the detailed origin of the structure. The agreement is satisfactory and the remaining differences are due in part to the facts that the calculation refers to a temperature of $0 \mathrm{~K}$, whereas the sample in the experiment was at $320 \mathrm{~K}$ and is thus affected by hot bands, and to the use of the harmonic approximation in the calculation. The calculation provides interesting insights: the origin of the band (the $0-0$ transition) has nearly no intensity because of the very large difference between the NO bond length in the neutral and ion states (1.27 versus $1.17 \AA$ ). We also 
Table 1. Summary of the results of the calculations with def2-TZVPD basis as described in Section 2.2. $f_{\mathrm{L}}$ is the oscillator strength and $\Delta\left\langle R^{2}\right\rangle$ (a.u.) is a measure of the spatial extent of the wave function in the excited state (see Section 3.2).

\begin{tabular}{|c|c|c|c|c|c|c|c|c|c|c|}
\hline \multirow[b]{2}{*}{ \# } & \multicolumn{4}{|c|}{ TDDFT/PBE0 } & \multicolumn{3}{|c|}{$\mathrm{CC} 2$} & \multicolumn{2}{|c|}{ NEVPT2 $^{\mathrm{a}}$} & \multirow[t]{2}{*}{ exp. } \\
\hline & State symmetry & Type & $E(\mathrm{eV})$ & $f_{\mathrm{L}}$ & $E(\mathrm{eV})$ & $f_{\mathrm{L}}$ & $\Delta\left\langle R^{2}\right\rangle$ & $E(\mathrm{eV})$ & $f_{\mathrm{L}}$ & \\
\hline \multicolumn{11}{|c|}{$S=1 / 2$} \\
\hline 1 & $1 a^{\prime \prime}$ & $n \rightarrow \pi^{*}$ & 2.78 & 0.0002 & 2.84 & 0.0003 & 0.1 & 2.71 & 0.0001 & 2.55 \\
\hline 2 & $2 a^{\prime}$ & $\pi^{*} \rightarrow 3 s$ & 4.83 & 0.0029 & 4.60 & 0.0033 & 14.1 & 5.63 & 0.0015 & \\
\hline 3 & $3 a^{\prime}$ & val. $^{\mathrm{b}}$ & 5.31 & 0.0252 & 5.73 & $0.0391\left(5 a^{\prime}\right)$ & 3.3 & 6.12 & 0.0198 & 4.96 \\
\hline 4 & $2 a^{\prime \prime}$ & $\pi^{*} \rightarrow 3 p$ & 5.35 & 0.0001 & 5.14 & 0.0001 & 18.3 & & & \\
\hline 5 & $4 a^{\prime}$ & $\pi^{*} \rightarrow 3 p$ & 5.41 & 0.0020 & 5.11 & $0.0009\left(3 a^{\prime}\right)$ & 13.9 & & & \\
\hline 6 & $5 a^{\prime}$ & $\pi^{*} \rightarrow 3 p$ & 5.48 & 0.0190 & 5.15 & $0.0061\left(4 a^{\prime}\right)$ & 12.0 & & & \\
\hline 7 & $6 a^{\prime}$ & & 5.92 & 0.0018 & 5.84 & 0.0044 & 13.7 & & & \\
\hline 8 & $3 a^{\prime \prime}$ & & 6.16 & 0.0076 & 5.95 & 0.0062 & 19.9 & & & \\
\hline 9 & $7 a^{\prime}$ & & 6.16 & 0.0012 & & & & & & \\
\hline 10 & $8 a^{\prime}$ & & 6.22 & 0.0002 & & & & & & \\
\hline 11 & $4 a^{\prime \prime}$ & & 6.23 & 0.0013 & 6.30 & $0.0008\left(5 a^{\prime \prime}\right)$ & 13.9 & & & \\
\hline 12 & $5 a^{\prime \prime}$ & & 6.27 & 0.0052 & 6.03 & $0.0079\left(4 a^{\prime \prime}\right)$ & 18.9 & & & \\
\hline 13 & $9 a^{\prime}$ & & 6.34 & 0.0125 & & & & & & \\
\hline \multicolumn{11}{|c|}{$S=3 / 2$} \\
\hline 14 & $1 a^{\prime \prime}$ & & 6.42 & (SCF) & & & & $8.95^{\mathrm{c}}$ & & \\
\hline 15 & $1 a^{\prime}$ & & 7.80 & (SCF) & & & & $9.78^{\mathrm{c}}$ & & \\
\hline \multirow[t]{2}{*}{$\mathrm{IP}^{\mathrm{d}}$} & (SOMO) & & 7.46 & (vert.) & & & & & & $7.27^{\mathrm{e}}$ \\
\hline & & & 6.92 & (adiab.) & & & & & & $6.89^{\mathrm{e}}$ \\
\hline
\end{tabular}

${ }^{a}$ Doublet, four roots, $\operatorname{CAS}(5,6)$.

${ }^{\mathrm{b}}$ Two-configurational valence-Rydberg mixed state composed of $\pi \rightarrow \pi^{*}(\beta)$ and $\pi^{*}(\mathrm{SOMO}) \rightarrow 3 p$ single excitations.

${ }^{\mathrm{c}}$ State-averaged doublet/quartet; four/four roots; CAS $(5,6)$, def2-TZVP.

${ }^{\mathrm{d}}$ TZVP basis set used in the PBE0 computations.

${ }^{\mathrm{e}}$ Taken as the first shoulder and the band maximum, respectively. Section 3.1 discusses the limits of this interpretation.

note a strong excitation of a $60 \mathrm{~cm}^{-1}$ vibration corresponding to the pyramidalisation of the $\mathrm{ONR}_{2}$ group where the entire $\mathrm{N}=\mathrm{O}$ group vibrates 'out of plane' of the molecule. The pyramidalisation around the $\mathrm{N}$ atom is also prominent around $650 \mathrm{~cm}^{-1}$, driven by the fact that the cation is planar around the $\mathrm{N}$-atom, whereas the neutral molecule is pyramidal. The first shoulder of the experimental spectrum thus does not correspond to the adiabatic ionisation energy as could naively be assumed, but to the maximum of the pyramidalisation progression. Note that the experimental and calculated data in Figure 3 are on the same energy scale, without any empirical adjustment, indicating an excellent accuracy of the calculated ionisation energy.

\subsection{Neutral molecule}

Experimental indication of the excitation energies is given by electron energy-loss spectra (EELS) in Figure 1, recorded under conditions favouring the allowed $\left(\theta=5^{\circ}\right.$, $\left.E_{r}=20 \mathrm{eV}\right)$ and the forbidden $\left(\theta=135^{\circ}, E_{r}=0.4 \mathrm{eV}\right)$ transitions. Both spectra show the ${ }^{2}\left(n, \pi^{*}\right)$ band at 2.55 $\mathrm{eV}$, in agreement with a ultraviolet (UV) absorption spectrum $[47,48]$. This band is solvatochromic, with vertical transitions (assumed as band maxima) varying smoothly with decreasing solvent polarity from $2.9 \mathrm{eV}$ in water to $2.60 \mathrm{eV}$ in cyclohexane $[47,49]$ so that the present vacuum value is an extrapolation of the solvatochromic trend. Our calculated values given in Table 1 are in good agreement, but slightly above, the experimental value. The very small values of the cross section, indicated in Figure 1, reflect the very small calculated oscillator strength. (The count rates were correspondingly low: $0.8 \mathrm{c} / \mathrm{s}$ at the peak of the $5 \mathrm{eV}$ band in the $E_{r}=20 \mathrm{eV}$ spectrum - requiring $130 \mathrm{~h}$ of acquisition time.) The sharp peak at $\Delta E=0.72 \mathrm{eV}$ in the bottom trace of Figure 1 is due to excitation of the first overtone of the $\mathrm{C}-\mathrm{H}$ stretch vibration.

The next prominent feature is a band in the $4-6 \mathrm{eV}$ range, peaking at $4.96 \mathrm{eV}$ in the $\theta=5^{\circ}$ spectrum. (A UV absorption band was reported at $5.12 \mathrm{eV}$ for an acetonitrile solution of TEMPO [8].) The shape is different in the $\theta=135^{\circ}$ spectrum, indicating the presence of several states, some being excited by non-dipole mechanisms at $135^{\circ}$. The $f_{\mathrm{L}}$ values calculated by TDDFT and CC2 (Table 1) indicate that this band is dominated by the $3 a^{\prime}$ valence-Rydberg mixed state composed of $\pi \rightarrow \pi^{*}(\beta)$ and $\pi^{*}(\mathrm{SOMO}) \rightarrow 3 \mathrm{p}$ excitations. The Rydberg character of a state can be judged from the $\Delta\left\langle R^{2}\right\rangle$ (difference of the electronic spatial extent between the ground and the excited states) values in Table 1, which indicate how much larger is the electron density in the excited state than in the ground state. The purely valence ${ }^{2}\left(n, \pi^{*}\right)$ state has $\Delta\left\langle R^{2}\right\rangle$ of nearly zero, while the clearly Rydberg states have values above 12 a.u. The $3 a^{\prime}$ state is thus closer to a valence than a Rydberg state. The $5 a^{\prime 2}\left(\pi^{*}, 3 p\right)$ state is predicted to also make a 


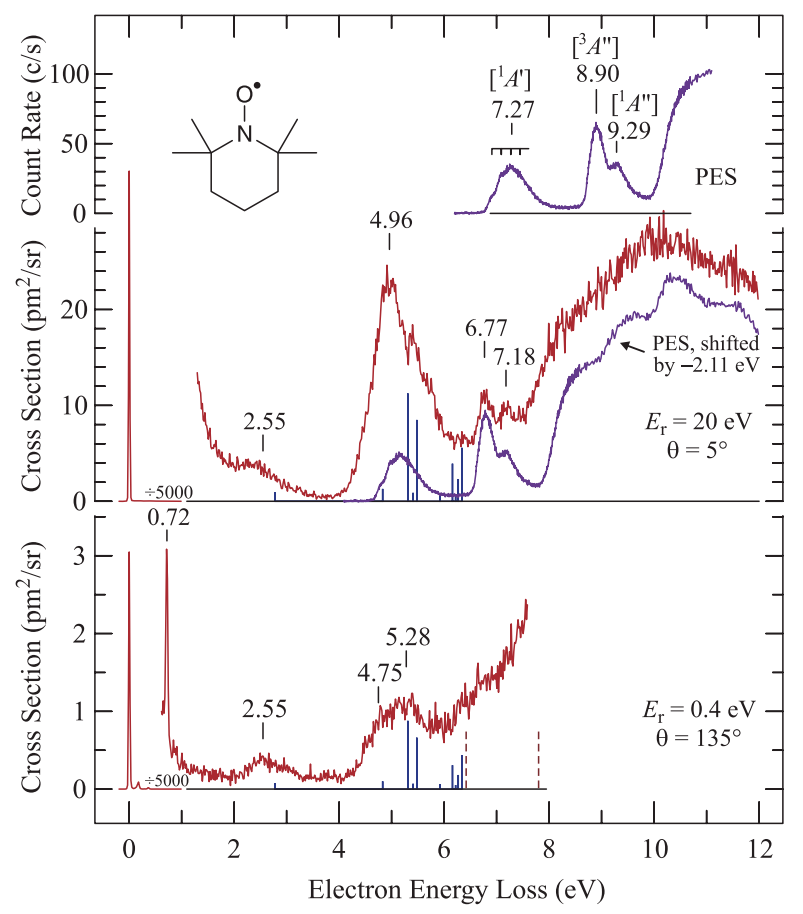

Figure 1. Top trace: HeI photoelectron spectrum. Bottom two traces: EELS recorded under conditions favouring spin-allowed $(\theta$ $\left.=5^{\circ}, E_{r}=20 \mathrm{eV}\right)$ and spin-forbidden $\left(\theta=135^{\circ}, E_{r}=0.4 \mathrm{eV}\right)$ transitions. A horizontally shifted photoelectron spectrum is shown under the $E_{r}=20 \mathrm{eV}$ EEL spectrum to illustrate the similarity of shapes. The vertical solid (blue) bars indicate the results of the PBE0 theory for the spin-allowed transitions and the dashed (orange) bars for the spin-forbidden transitions. [The bar for the ${ }^{2}\left(n, \pi^{*}\right)$ state at $2.78 \mathrm{eV}$ is shown 10 times vertically expanded.]

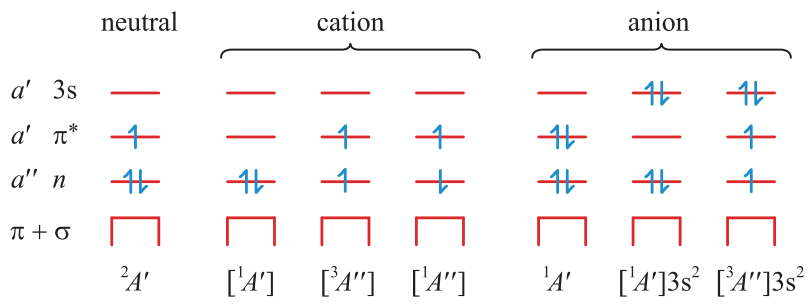

Figure 2. Representative electron configurations of TEMPO, and its cation and anion. The square brackets enclose the designation of the cation core.

significant contribution to the $4.96 \mathrm{eV}$ band. The $3 a^{\prime}$ and $5 a^{\prime}$ states are calculated only slightly above the observed peak, by about the same amount as the ${ }^{2}\left(n, \pi^{*}\right)$ state - the agreement is good. A group of states with significant $f_{\mathrm{L}}$ values are calculated just above $6 \mathrm{eV}$ and are reflected in the $5^{\circ}$ spectrum by the clearly non-zero cross section in the 6-6.4 eV range.

The EELS-PES comparison in Figure 1 reveals a striking similarity of the neutral excited states to the cationic states. The 4.96 band is revealed by the calculations to be largely due to low ( $3 s$ and $3 p$ ) Rydbergs with the $\left[{ }^{1} A^{\prime}\right]$ ground state cation core. By analogy, the 6.77 and $7.18 \mathrm{eV}$

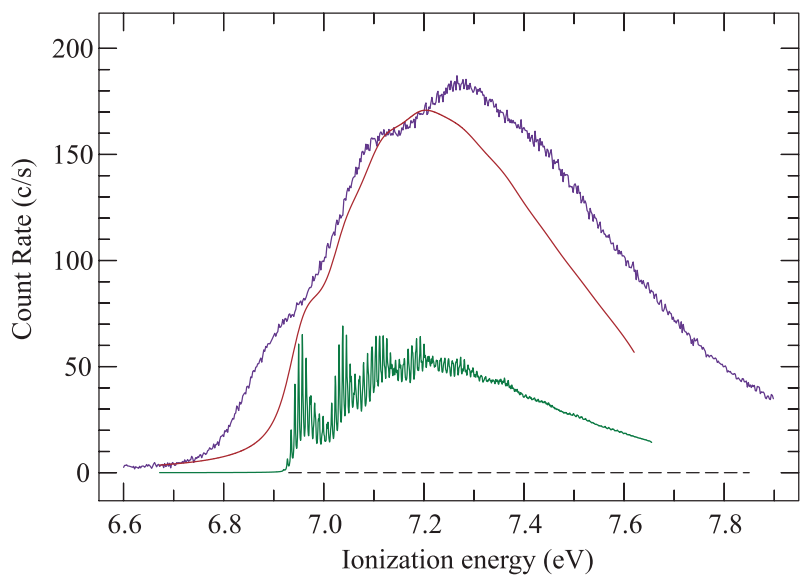

Figure 3. HeI photoelectron spectrum of the first band (curve with statistical noise), compared to the calculated FC profile, convoluted with Lorentzians of $10 \mathrm{~cm}^{-1}$ and $250 \mathrm{~cm}^{-1}$ widths. The origin of the FC profile is put into the calculated adiabatic ionisation energy of $6.92 \mathrm{eV}$, without any empirical shift.

bands in the EELS can be assigned to Rydbergs with the $\left[{ }^{3} A^{\prime \prime}\right]$ and $\left[{ }^{1} A^{\prime \prime}\right]$ excited state cation cores. This PESEELS analogy appears to go even further, the $7.5-12 \mathrm{eV}$ 'mountain' in the EELS closely mimics the 'mountain' of unresolved bands in the shifted PES and may thus be assigned to Rydbergs with cores ionised from deeper valence orbitals.

It is remarkable that the PES needs to be shifted by only $-2.11 \mathrm{eV}$ to correspond to the Rydberg states - indicating small-term energy (ionisation energy minus the excitation energy) of only $2.11 \mathrm{eV}$. Following the discussion of Robin $[50,51]$, this is due to the large alkyl body of TEMPO, which 'protects' the charge on the $\mathrm{N}=\mathrm{O}$ group, hindering the approach of the Rydberg electron and thus reducing the attraction between the Rydberg electron and the ion core. For comparison, the term energy of an 'unprotected' $\mathrm{C}=\mathrm{C}$ double bond is around $3.5 \mathrm{eV}$ [51], that of bicyclohexylidene, also with large alkyl groups, is $2.6 \mathrm{eV}$ [52], so that the protecting effect in TEMPO goes even beyond that in bicyclohexylidene.

The quantum chemical calculations of the excitation energies with three conceptually different methods (timedependent DFT, time-dependent wave function theory, spinrestricted multi-reference perturbation theory) show good agreement with each other also regarding the qualitative character of the states involved.

\subsection{Negative ion}

Nuclear relaxation on the potential surfaces of the shortlived ( $\sim$ ps) states of the negative ion (resonances) leads to $\mathrm{VE}$ and the corresponding cross sections thus represent an efficient means of detecting resonances [53]. This method detects preferentially the shape resonances, anions with a 


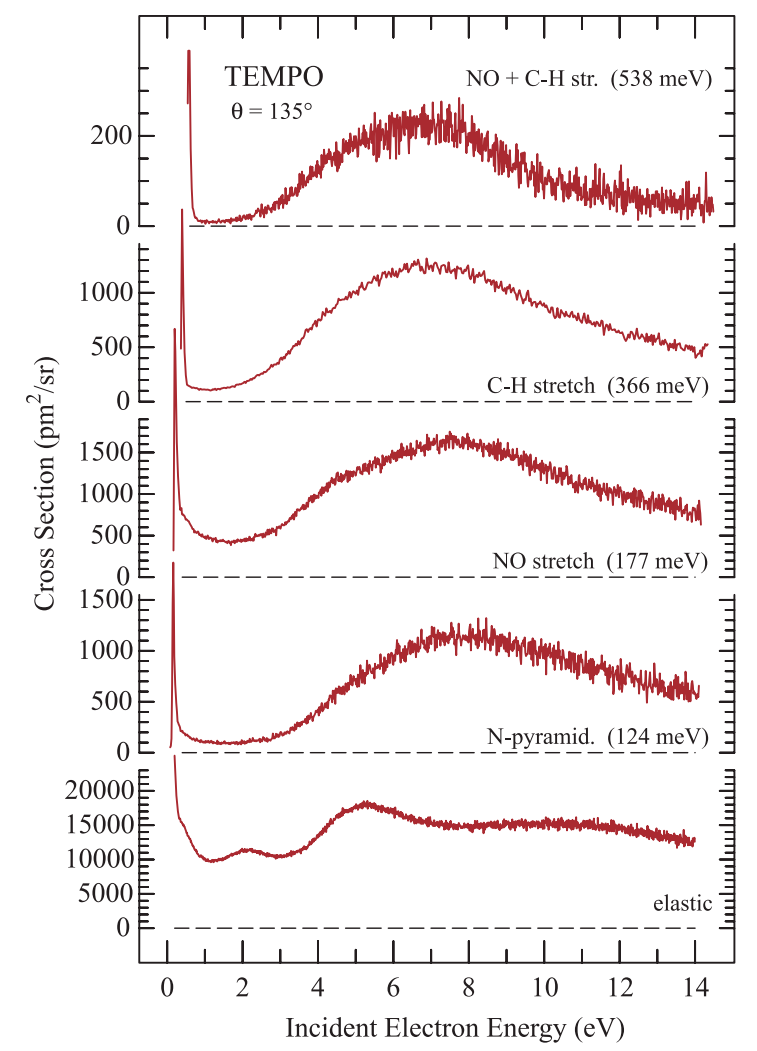

Figure 4. Elastic and VE cross sections.

temporary occupation of a normally empty orbital around a neutral core in its electronic ground states. The cross sections in Figure 4 show that electron collisions with TEMPO excite primarily the $\mathrm{N}-\mathrm{O}$ stretch, the $\mathrm{C}-\mathrm{H}$ stretch, and the $\mathrm{N}$-pyramidalisation vibrations. (The $\mathrm{N}-\mathrm{O}$ stretch vibration was reported at $166 \mathrm{meV}$ [54], but our B3LYP calculations indicate four vibrations with a pronounced $\mathrm{N}-\mathrm{O}$ stretch motion in the range $170-180 \mathrm{meV}$ and more than one of these vibrations appear to be excited by electron impact, leading to a broadened EEL band peaking at $177 \mathrm{meV}$. Our cross section applies to this entire band.) The dominant feature in all three VE cross sections is an extremely broad band extending from about $3 \mathrm{eV}$ to beyond $14 \mathrm{eV}$. This type of band is found in all polyatomic organic compounds, in particular the unsaturated [55] and saturated hydrocarbons [56], and is assigned to resonances with temporary electron capture in $\sigma^{*}(\mathrm{C}-\mathrm{C})$ and $\sigma^{*}(\mathrm{C}-\mathrm{H})$ orbitals. The extreme width of the band is caused in part by the very short lifetime (given by the very fast autodetachment of the extra electron) via Heisenberg's uncertainty relation, and in part by the presence of unresolved overlapping resonances. The presence of several distinct overlapping $\sigma^{*}$ resonances (rather than only one extremely wide resonance) in the 2 $12 \mathrm{eV}$ range of saturated alkyl moieties was revealed by the studies on cyclopropane [56,57], where high symme- try makes the resonances narrower, so that they can be distinguished individually. We conclude that a number of resonances of a similar type is also present in TEMPO, but they overlap to such a degree that they cannot be recognised individually.

The narrow peaks at thresholds are evidence for dipolebound anions of TEMPO - the vibrational Feshbach resonances [58]. The dipole moment of TEMPO [calculated at the B3LYP 6-311G $(2 d, p)$ level] is $2.9 \mathrm{D}$, which is above the critical value for dipole binding.

Apart from the above two features, VE is also observed in the $0-2 \mathrm{eV}$ range, in particular for the $\mathrm{N}-\mathrm{O}$ stretch mode, and is indicative of the ${ }^{1} A^{\prime}$ state with a temporary double occupation of the $\pi^{*}$ orbital (see Figure 2). The signal rises with decreasing energy, without building a band with a peak, indicating that the state is either slightly below or slightly above zero energy, and the electron is either slightly bound or slightly unbound. Our experiment cannot, unfortunately, distinguish between these two possibilities. Calculations [B3LYP/6-311++G $(2 d, p)]$ indicate the valence state of the anion to be adiabatically (harmonic zero-point energy corrected) slightly bound (by $0.14 \mathrm{eV}$ ) and vertically slightly unbound, by $0.3 \mathrm{eV}$, that is, a vertical electron attachment energy to be about $0.3 \mathrm{eV}$. This prediction is compatible with the experiment. The situation is complicated by the fact that the valence anion may mix with the dipole-bound state, although calculations (comparison of the equilibrium geometries of the valence anion and the neutral molecule) indicate that the geometries of the dipole-bound anion and the valence anion are quite different, similarly to the nitromethane case [59-61].

The visibility of the $\pi^{*}$ resonance in the NO stretch cross section is surprisingly modest when compared with the $\pi^{*}$ resonances in, for example, ethene $[55,62]$ or formic acid [63]. The cross section for exciting the $\mathrm{C}=\mathrm{C}$ and $\mathrm{C}=\mathrm{O}$ stretch vibrations have a clearly visible peak at the energy of the $\pi^{*}$ resonance in these molecules. Comparison with Refs. [62] and [63] reveals that the TEMPO cross section in the $\pi^{*}$ region $\left(700 \mathrm{pm}^{2}\right.$ at $0.5 \mathrm{eV}$ and $135^{\circ}$, see Figure 4 ) is not substantially smaller in magnitude than the cross sections (both also at $135^{\circ}$ ) at the top of the $\pi^{*}$ resonances in ethene $\left(1000 \mathrm{pm}^{2}\right.$ at $1.85 \mathrm{eV}$ [62] $)$ or formic acid $\left(600 \mathrm{pm}^{2}\right.$ at $1.9 \mathrm{eV}$ [63]); the difference is that the TEMPO cross section does not form a clear band. This is, on the one hand, due to the fact that the $\sigma^{*}$ band is much stronger $\left(1700 \mathrm{pm}^{2}\right.$ at $\left.7.5 \mathrm{eV}\right)$, and thus overlaps with the $\pi^{*}$ band more, in TEMPO than in ethene $\left(250 \mathrm{pm}^{2}\right.$ at $\left.8 \mathrm{eV}\right)$ or formic acid, presumably because of the many more $\mathrm{C}-\mathrm{C}$ and $\mathrm{C}-\mathrm{H}$ bonds in TEMPO and thus many more $\sigma^{*}$ orbitals. On the other hand, the fact that the vertical attachment energy in TEMPO is low, so that the peak of the $\pi^{*}$ band is near $0 \mathrm{eV}$, explains why no $\pi^{*}$ peak appears in the cross section. (The angular distributions are likely to be slightly different for the three compounds, but this does not change the qualitative argument.) 
We further observe a fairly strong (in comparison to ethene and formic acid) production of very slow $(\sim 0$ $100 \mathrm{meV}$, peaking at $0 \mathrm{eV}$ ) electrons in the $\pi^{*}$ resonance region in TEMPO. It reveals a process whereby the highest vibrational levels attainable with a given incident electron energy are excited, without pronounced specificity for any particular mode, and a near-zero energy electron is then released (the 'unspecific' VE, observed for many large molecules [64]). The prominence of this process is linked to the large size of the TEMPO molecule and the ensuing large number of vibrational modes, forming a dense quasicontinuum into which the system can go, even though the initial relaxation on the resonant potential surface is primarily in the direction of the $\mathrm{N}-\mathrm{O}$ stretch. The process is thus related to intramolecular vibrational redistribution . The detachment of very low energy electrons is slow and the highly vibrationally excited anions must thus have lifetimes much longer than the characteristic ps lifetimes of resonances. We observed a preliminary indication of this in the form of ion-molecule reactions in the DEA spectrometers at higher pressures and in the $0-1 \mathrm{eV}$ energy range.

The elastic cross section is given for completeness at the bottom of Figure 4. Elastic cross section is generally not the preferred means for observing resonances because it is dominated by direct processes which mask the resonant contribution (in contrast to the VE cross section which is generally dominated by resonant processes). Resonances are visible in the elastic cross section of some molecules, though, the lowest resonances in $\mathrm{N}_{2}[53,65]$ or ethene [62]) being prominent examples. In this sense, it is interesting to observe that a weak shoulder is observed in the elastic cross section in Figure 4 at about $0.5 \mathrm{eV}$ where the vertical $\pi^{*}$ resonance is expected.

\subsection{Dissociative electron attachment}

In this final section, we present a chemical change induced by free electrons, for whose understanding the above spectroscopic information will prove helpful. This paper will be limited to the strongest fragmentation process, yielding the $\mathrm{O}^{-}$fragment, the cross section for which is shown in Figure 5. Other fragments, including $\mathrm{H}^{-}$, were also observed and will be presented in a subsequent publication. To facilitate the assignment of the resonances responsible for the DEA, its spectrum is compared to the EELS showing the potential parent states, the PES showing the potential grandparent states, and a representative VE cross section, giving an overview of the shape resonances.

The shape resonances revealed by the VE spectra are clearly not causing the DEA because the shapes, particularly the widths, of the VE and the DEA bands are very different. On the other hand, the lower DEA band corresponds to the EEL band both in energy and in shape, pointing to a core-excited resonance being at the origin of the DEA, with

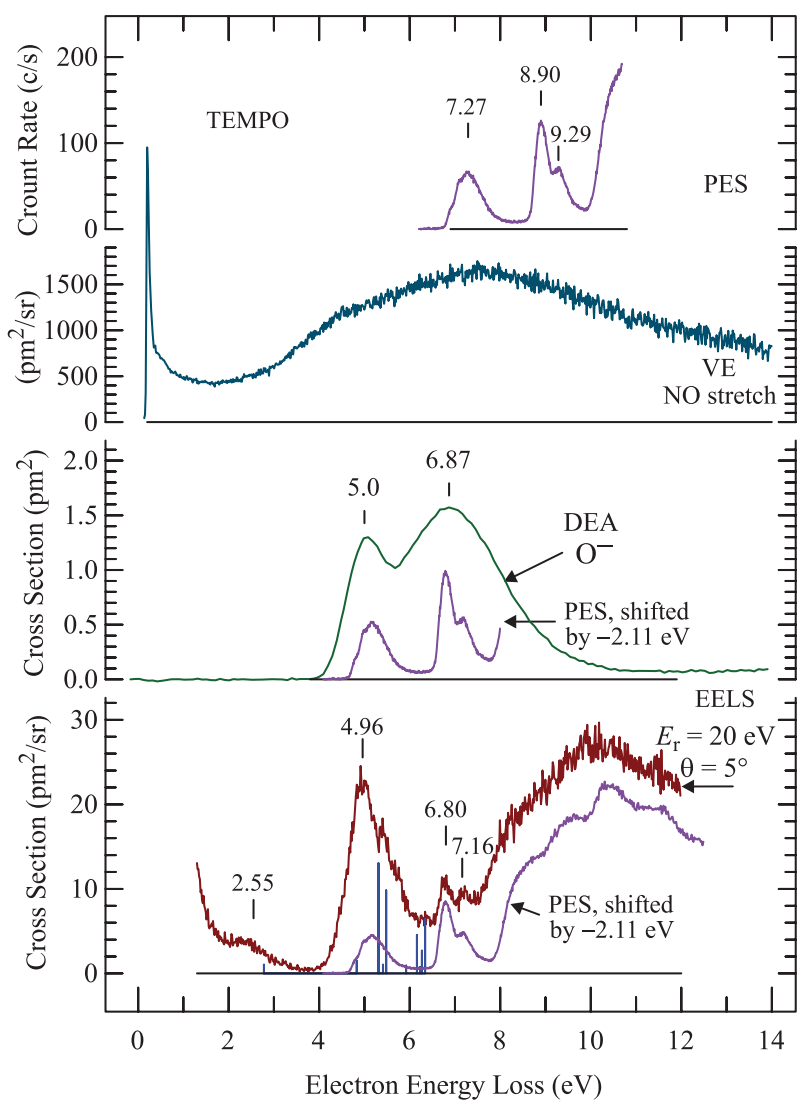

Figure 5. Dissociative electron attachment cross section (labelled DEA), compared to EELS, VE spectra and PESs.

one (or several) of the states within the 4.96 EEL band as parents.

Two situations are quite common in DEA. In the first, a DEA band is observed about $0.4 \mathrm{eV}$ below a parent Rydberg state and is assigned to a Feshbach resonance, with a doubly occupied Rydberg-like orbital around a positive ion core. Such bands are omnipresent in saturated compounds such as alcohols and amines $[66,67]$. The second case is often found in unsaturated compounds, where valence excited states lie well below Rydberg states, and has a DEA band nearly coinciding with the lowest singlet excited state, assigned to a resonance where the incoming electron is temporarily captured in a diffuse orbital around a valence excited core. Systematic evidence for such states was collected by the group of Khvostenko [68]. We have observed this type of resonances in many compounds in the past, for example, in $\mathrm{CS}_{2}$ [69] and chlorobenzene [70].

In the present case, it is difficult to distinguish between the two possibilities, because the valence and the Rydberg excited states which serve as potential parents lie very close together. It may be argued that the Feshbach resonances are generally about $0.4 \mathrm{eV}$ below their parent Rydberg state (i.e. the parent Rydberg state has an electron affinity of about $0.4 \mathrm{eV})$, whereas the present DEA band practically 
coincides with the parent-state band. However, the reduced binding could be due to the large alkyl part of the molecule, in the same way as it reduces the binding of a Rydberg electron to the cation to form a Rydberg state, discussed in Section 3.2

The second DEA band at $6.87 \mathrm{eV}$ corresponds in energy to the pair of the second and third photoelectron bands, indicating that the resonance involved in this DEA band could have them as parents. The similarity of the grandparent and the daughter DEA bands is less pronounced in this case; however, the DEA band is much broader. The proposition that higher lying DEA bands should be assigned to core excited resonances with holes in deeper lying orbitals is not new - it was proposed in the case of water by Jungen et al. [71] and more generally by Robin [51].

Note that the various magnitudes in Figure 5 cannot be compared directly - the DEA cross section is integral, integrated over all angles into which the fragment can depart, with the unit $\mathrm{pm}^{2}$. The VE and EEL cross sections are differential, for a specific scattering angle $\left(135^{\circ}\right)$, with the unit $\mathrm{pm}^{2} / \mathrm{sr}$. A coarse estimate of the integral VE and EEL cross sections, under an assumption of isotropic scattering, is obtained by multiplying the differential cross section by $4 \pi$. Figure 5 thus shows that DEA in TEMPO is not an efficient process, the cross section is much smaller than that for VE, and smaller than that for electronic excitation.

\section{Conclusions}

The combination of several electron-spectroscopic techniques is a powerful tool for the study of electronic structure, including the states of the neutral molecule, and its cation and anion. The emphasis is not on high resolution, but on an overview including even highly excited states. The knowledge gained on the cation and the neutral molecules is useful in assigning the anion states (resonances) responsible for dissociative electron attachment. TEMPO offers the opportunity to study whether there are any general 'unpaired electron effects' in electron collisions. All the findings are quite 'normal', however, similar to those in closed-shell molecules, perhaps with the exception of the obvious greater stability of the ground-state anion, with electron capture into the singly occupied molecular orbital (SOMO), when compared to a similar alkene or carbonyl compound, with electron capture into the lowest unoccupied molecular orbital. The quantum chemical methods used, in particular TDDFT/PBE0, reproduced the observed excitation energies with a good accuracy, of the order of 0.2-0.4 $\mathrm{eV}$. It can be concluded that the combination of high-level spectroscopic techniques with accurate quantum chemical calculations offers a great perspective to gain insights into the complicated electronic structure of radicals and their excited states.

\section{Acknowledgements}

This work was supported by projects No. 200020-144367/1 and PZ00P2_132357/1 of the Swiss National Science Foundation and the SFB 813 ('Chemistry at Spin Centers') of the German Science Foundation.

\section{References}

[1] T.M. Maddern, L.R. Hargreaves, J.R. Francis-Staite, M.J. Brunger, S.J. Buckman, C. Winstead, and V. McKoy, Phys. Rev. Lett. 100(6), 063202 (2008).

[2] M. Allan, Phys. Rev. Lett. 93, 063201 (2004).

[3] E.G. Rozantsev, Free Nitroxyl Radicals (Plenum Press, New York-London, 1970).

[4] G.I. Likhtenshtein, J. Yamauchi, S. Nakatsuji, A.I. Smirnov, and R. Tamura, Nitroxides (Wiley-VCH, Weinheim, 2008).

[5] H. Karoui, F. Le Moigne, O. Ouari, and P. Tordo, in Stable Radicals, edited by E.G. Hicks (J. Wiley \& Sons, Chichester, 2010), pp. 173-229.

[6] K. Nakahara, K. Oyaizu, and H. Nishide, Chem. Lett. 40, $222(2011)$

[7] T. Janoschka, M.D. Hager, and U.S. Schubert, Adv. Mater. 24, 6397 (2012).

[8] K. Nakahara, S. Iwasa, J. Iriyama, Y. Morioka, M. Suguro, M. Satoh, and E.J. Cairns, Electrochim. Acta 52, 921 (2006).

[9] J.P. Blinco, J.L. Hodgson, B.J. Morrow, J.R. Walker, G.D. Will, M.L. Coote and S.E. Bottle, J. Org. Chem. 73, 6763 (2008)

[10] F. Kato, N. Hayashi, T. Murakami, C. Okumura, K. Oyaizu, and H. Nishide, Chem. Lett. 39, 464 (2010).

[11] C. Buhmester, L.M. Moshurchak, R.L. Wang, and J.R. Dahn, J. Electrochem. Soc. A 153, 1800 (2006).

[12] F. Lin, D. Pei, W. He, Z. Huang, Y. Huang, and X. Guo, J. Mater. Chem. 22, 11801 (2012).

[13] T. Kusamoto, S. Kume, and H. Nishihara, Angew. Chem. Int. Ed. 49, 529 (2010).

[14] M. Lee, M. Kang, B. Moon, and H.B. Oh, Analyst 134, 1706 (2009).

[15] D.H. Solomon, J. Polym. Sci., Part A: Polym. Chem. 43, 5748 (2005).

[16] D. Bertin, D. Gigmes, S.R.A. Marque, and P. Tordo, Chem. Soc. Rev. 40, 2189 (2011)

[17] D. Gigmes and S.R.A. Marque, in Encyclopedia of Radicals in Chemistry, Biology and Materials, edited by $\mathrm{Ch}$ Chatgilialoglu and A. Studer (J. Wiley \& Sons, Chichester, 2012), Vol. 4, pp. 1813-1850.

[18] A.J.P. Cardenas, B.J. Culotta, T.H. Warren, S. Grimme, A. Stute, R. Fröhlich, G. Kehr, and G. Erker, Angew. Chem. Int. Ed. 50, 7567 (2011)

[19] O. May, J. Fedor, and M. Allan, Phys. Rev. A 80, 012706 (2009).

[20] J. Fedor, O. May, and M. Allan, Phys. Rev. A 78, 032701 (2008).

[21] M. Stepanović, Y. Pariat, and M. Allan, J. Chem. Phys. 110, 11376 (1999).

[22] R. Dressler and M. Allan, Chem. Phys. 92, 449 (1985).

[23] M. Allan, J. Phys. B: At. Mol. Opt. Phys. 40, 3531 (2007).

[24] M. Allan, Phys. Rev. A 81, 042706/1 (2010).

[25] J.P. Perdew, K. Burke, and M. Ernzerhof, Phys. Rev. Lett. 77, 3865 (1996); erratum Phys. Rev. Lett. 78, 1396 (1997).

[26] C. Adamo and V. Barone, J. Chem. Phys. 110, 6158 (1999).

[27] A. Schäfer, C. Huber, and R. Ahlrichs, J. Chem. Phys. 100, 5829 (1994).

[28] F. Weigend and R. Ahlrichs, Phys. Chem. Chem. Phys. 7 , 3297 (2005). 
[29] S. Grimme, J. Antony, S. Ehrlich, and H. Krieg, J. Chem. Phys. 132, 154104 (2010).

[30] S. Grimme, S. Ehrlich, and L. Goerigk, J. Comput. Chem. 32, 1456 (2011)

[31] M.E. Casida, Time-Dependent Density Functional Response Theory for Molecules, Recent Advances in Density Functional Methods, edited by D.P. Chong, (World Scientific, Singapore, 1995), 1, pp. 155-192.

[32] M.A.L. Marques, C.A. Ulrich, F. Nagueira, A. Rubio, K. Burke, and E.K.U. Gross, editors, Time-Dependent Density Functional Theory, Lecture Notes Phys. 706 (Springer, Berlin-Heidelberg, 2006).

[33] O. Christiansen, H. Koch, and P. Jørgensen, Chem. Phys. Lett. 243, 409 (1995).

[34] C. Hättig and F. Weigend, J. Chem. Phys. 113, 5154 (2000).

[35] TURBOMOLE 6.3: R. Ahlrichs, M.K. Armbruster, M. Bär, H.-P. Baron, R. Bauernschmitt, N. Crawford, P. Deglmann, M. Ehrig, K. Eichkorn, S. Elliott, F. Furche, F. Haase, M. Häser, C. Hättig, A. Hellweg, H. Horn, C. Huber, U. Huniar, M. Kattannek, C. Kölmel, M. Kollwitz, K. May, P. Nava, C. Ochsenfeld, H. Öhm, H. Patzelt, D. Rappoport, O. Rubner, A. Schäfer, U. Schneider, M. Sierka, O. Treutler, B. Unterreiner, M. von Arnim, F. Weigend, P. Weis, and H. Weiss. Universität Karlsruhe 2012. See also: http://www.turbomole.com.

[36] D. Rappoport and F. Furche, J. Chem. Phys. 133, 134105 (2010).

[37] M. Dierksen and S. Grimme, J. Chem. Phys. 120, 3544 (2004).

[38] M. Dierksen and S. Grimme, J. Phys. Chem. A 108, 10225 (2004).

[39] C. Angeli, R. Cimiraglia, S. Evangelisti, T. Leininger, and J.P. Malrieu, J. Chem. Phys. 114, 10252 (2001).

[40] F. Neese, ORCA - An ab initio, Density Functional and Semiempirical Program Package, Ver. 2.9 (Rev 0) (Max Planck Institute for Bioinorganic Chemistry, Germany, 2011).

[41] F. Neese, WIREs Comput. Mol. Sci. 2, 73 (2012).

[42] F. Neese, J. Am. Chem. Soc. 128, 10213 (2006).

[43] A.A. Granovsky, Firefly version 8.0.0 <http://classic.chem .msu.su/gran/firefly/index.html>.

[44] M.W. Schmidt, K.K. Baldridge, J.A. Boatz, S.T. Elbert, M.S. Gordon, J.H. Jensen, S. Koseki, N. Matsunaga, K.A. Nguyen, S. Su, T.L. Windus, M. Dupuis, and J.A. Montgomery, J. Comput. Chem. 14, 1347 (1993).

[45] M.J. Frisch, G.W. Trucks, H.B. Schlegel, G.E. Scuseria, M.A. Robb, J.R. Cheeseman, G. Scalmani, V. Barone, B. Mennucci, G.A. Petersson, H. Nakatsuji, M. Caricato, X. Li, H.P. Hratchian, A.F. Izmaylov, J. Bloino, G. Zheng, J.L. Sonnenberg, M. Hada, M. Ehara, K. Toyota, R. Fukuda, J. Hasegawa, M. Ishida, T. Nakajima, Y. Honda, O. Kitao, H. Nakai, T. Vreven, J.A. Montgomery, Jr., J.E. Peralta, F. Ogliaro, M. Bearpark, J.J. Heyd, E. Brothers, K.N. Kudin, V.N. Staroverov, R. Kobayashi, J. Normand, K. Raghavachari, A. Rendell, J.C. Burant, S.S. Iyengar, J. Tomasi, M. Cossi, N. Rega, J.M. Millam, M. Klene, J.E. Knox, J.B. Cross, V. Bakken, C. Adamo, J. Jaramillo, R.
Gomperts, R.E. Stratmann, O. Yazyev, A.J. Austin, R. Cammi, C. Pomelli, J.W. Ochterski, R.L. Martin, K. Morokuma, V.G. Zakrzewski, G.A. Voth, P. Salvador, J.J. Dannenberg, S. Dapprich, A.D. Daniels, Ö. Farkas, J.B. Foresman, J.V. Ortiz, J. Cioslowski, and D.J. Fox, Gaussian 09 Revision A.02 (Gaussian, Inc., Wallingford CT, 2009).

[46] I. Novak, L.J. Harrison, B. Kovac, and L.M. Pratt, J. Org. Chem. 69, 7628 (2004).

[47] A. Janowski, I. Turowska-Tyrk, and P. Wrona, J. Chem. Soc. Perkin Trans. II p. 821 (1985).

[48] A. Moscatelli, M.F. Ottaviani, W. Adam, A. Buchachenko, S. Jockusch, and N.J. Turro, Helv. Chim. Acta 89, 2441 (2006).

[49] J. Laleveé, X. Allonas, and P. Jacques, THEOCHEM 767, 143 (2006)

[50] M.B. Robin, Higher Excited States of Polyatomic Molecules, Vol. 1 (Academic Press, New York, 1974).

[51] M.B. Robin, Higher Excited States of Polyatomic Molecules, Vol. 3 (Academic Press, Orlando, 1985).

[52] M. Allan, P.A. Schnyder, and M.B. Robin, J. Phys. Chem. 89, 4900 (1985).

[53] G.J. Schulz, Rev. Mod. Phys. 45, 423 (1973)

[54] L. Rintoul, A. Micallef, and S. Bottle, Spectrochim. Acta A 70, 713 (2008)

[55] I.C. Walker, A. Stamatovic, and S.F. Wong, J. Chem. Phys. 69, 5532 (1978).

[56] M. Allan and L. Andrić, J. Chem. Phys. 105, 3559 (1996).

[57] R. Čurík, P. Čársky, and M. Allan, Phys. Rev. A 86, 062709 (2012).

[58] H. Hotop, M.W. Ruf, M. Allan, and I.I. Fabrikant, Adv. At. Mol. Opt. Phys. 49, 85 (2003).

[59] R.N. Compton, H.S.J. Carman, C. Desfrancois, H. AbdoulCarmine, J.P. Schermann, J.H. Hendricks, S.A. Lyapustina, and K.H. Bowen, J. Chem. Phys. 105 (9), 3472 (1996).

[60] F. Lecomte, S. Carles, C. Desfrancois, and M.A. Johnson, J. Chem. Phys. 113(24), 10973 (2000).

[61] T. Sommerfeld, Phys. Chem. Chem. Phys. 4, 2511 (2002).

[62] M. Allan, C. Winstead, and V. McKoy, Phys. Rev. A 77, 042715 (2008).

[63] M. Allan, J. Phys. B: At. Mol. Opt. Phys. 39, 2939 (2006).

[64] M. Allan, J. Electron Spectrosc. Relat. Phenom. 48, 219 (1989).

[65] M. Allan, J. Phys. B: At. Mol. Opt. Phys. 38, 3655 (2005).

[66] T. Skalický and M. Allan, J. Phys. B 37, 4849 (2004).

[67] B.C. Ibănescu, O. May, A. Monney, and M. Allan, Phys. Chem. Chem. Phys. 9, 3163 (2007).

[68] V.I. Khvostenko, A.S. Vorob'yov, and O.G. Khvostenko, J. Phys. B: At. Mol. Opt. Phys. 23(12), 1975 (1990).

[69] R. Dressler, M. Allan, and M. Tronc, J. Phys. B: At. Mol. Phys. 20(2), 393 (1987).

[70] C. Bulliard, Ph. D. thesis, Département de Chimie, Université de Fribourg Suisse, 1994.

[71] M. Jungen, J. Vogt, and V. Staemmler, J. Phys. B 37, 49 (1979). 